\title{
QUALIDADE DE VIDA EM PACIENTES COM ENDOMETRIOSE: UM ESTUDO DE REVISÃO
}

\author{
Quality of life in patients with endometriosis: a review study \\ Calidad de vida de pacientes con endometriosis: un estudio de \\ revisión
}

Artigo de revisão

\section{RESUMO}

Objetivo: Avaliar a qualidade de vida $(\mathrm{QV})$ em pacientes com endometriose. Métodos: Realizou-se uma revisão bibliográfica na base de dados PubMed buscando artigos publicados nos últimos 10 anos utilizando os descritores quality of life and endometriosis e os seguintes filtros: artigos com abstract disponível, em humanos do sexo feminino, idioma inglês/ português e cujos termos constavam no título/abstract. A busca resultou em 147 artigos, dos quais, de acordo com os critérios de inclusão, restaram 12 para leitura. Resultados: Todos os artigos evidenciaram que as mulheres com endometriose apresentaram uma redução na QV, e cinco estudos associaram nível socioeconômico, intensidade da dor e hábitos saudáveis como fatores que influenciam a QV. No entanto, nenhum estudo propôs estratégias para a melhora da QV. A presente revisão evidencia os benefícios da abordagem fisioterapêutica, psicológica e da prática de atividade física em elevar a QV, como uma maneira de minimizar os sintomas da endometriose, em especial, a dor e os sintomas psicológicos. A avaliação da QV em endometriose poderia ser utilizada como um indicador prognóstico da melhora clínica. Conclusão: Mulheres com endometriose apresentam significativa redução em sua qualidade de vida, influenciada pelos aspectos físicos e psicológicos, entre outros.

Descritores: Endometriose; Qualidade de Vida; Promoção da Saúde; Doença Crônica.

\section{ABSTRACT}

Objective: This study aims to assess the quality of life (QoL) in patients with endometriosis. Methods: We conducted a literature review searching for articles published in the last 10 years in the PubMed database, using the descriptors 'quality of life' and 'endometriosis' and the following filters: in human female, articles with abstract available, English/Portuguese language, and containing the terms in the title/abstract. The search retrieved 147 articles, of which 12 remained for reading, according to the inclusion criteria. Results: All the articles pointed out that women with endometriosis showed a reduction in QoL, and five studies have linked socioeconomic status, severity of pain and healthy habits as factors that influence QoL. However, neither of the studies suggests strategies for the improvement of QoL. This review shows the benefits of the physiotherapeutic and psychological approach, and physical activity practice in raising $Q o L$ as a way to minimize the symptoms of endometriosis, especially pain and psychological symptoms. The assessment of QoL in endometriosis could be used as a prognostic indicator of clinical improvement. Conclusion: Women with endometriosis exhibit a significant reduction in their QoL, influenced by physical and psychological aspects, among others.

Descriptors: Endometriosis; Quality of Life; Health Promotion; Chronic Disease.

Recebido em: 05/05/2014 Revisado em: 19/05/2014 Aceito em: 12/06/2014

1) Universidade Federal do Triângulo Mineiro - UFTM - Uberaba (MG) - Brasil 


\section{RESUMEN}

Objetivo: Evaluar la calidad de vida (CV) de pacientes con endometriosis. Métodos: Se realizó una revisión bibliográfica en la base de datos PubMed con búsqueda de artículos publicados en los últimos 10 años utilizándose de los descriptores quality of life and endometriosis y los siguientes filtros: artículos con abstract disponible, en humanos del sexo femenino, idioma inglés/portugués y cuyos términos estaban en el título/abstract. La búsqueda resultó en 147 artículos, de los cuales, según los critérios de inclusión, restaron 12 para la lectura. Resultados: Todos los artículos evidenciaron que las mujeres con endometriosis presentaron reducción en la $C V$, y cinco estudios asociaron el nivel socioeconómico, la intensidad del dolor y hábitos saludables como factores que influyen en la $C V$. Sin embargo, ningún estudio ha propuesto estrategias para mejorar la CV. Esta revisión evidencia los beneficios del abordaje fisioterapéutico, psicológico y de la práctica de actividad fisica en mejorar la $C V$, como una manera de minimizar los sintomas de la endometriosis, en especial, el dolor y los síntomas psicológicos. La evaluación de la $\mathrm{CV}$ en endometriosis podría ser utilizada como un indicador pronóstico de la mejora clínica. Conclusión: Mujeres con endometriosis presentan reducción significativa de la calidad de vida debido, entre otros aspectos, a los físicos y psicológicos.

Descriptores: Endometriosis; Calidad de Vida; Promoción de la Salud; Enfermedad Crónica.

\section{INTRODUÇÃO}

A endometriose é uma condição ginecológica complexa, caracterizada por tecido endometrial em sítios extrauterinos. Seus sintomas incluem dor pélvica crônica (DPC), dismenorreia, dispareunia, disquezia, disúria e infertilidade ${ }^{(1)}$. Por se tratar de uma doença crônica, as pacientes com endometriose exibem redução na qualidade de vida (QV). Segundo a Organização Mundial de Saúde (OMS), QV é a percepção do indivíduo sobre sua posição na vida, no contexto da cultura e do sistema de valores em que vive e em relação aos seus objetivos, expectativas, padrões e preocupações ${ }^{(2)}$. De um ponto de vista relacionado à saúde, QV é um conceito multidimensional que engloba aspectos físicos, psicológicos e sociais relacionados a uma doença ou tratamento em particular ${ }^{(3)}$. Portanto, o conceito de QV é caracterizado pela subjetividade e multidimensionalidade.

Com relação a tratamento, diagnóstico, consultas, exames e procedimento cirúrgico, um estudo recente mostrou que o impacto econômico da endometriose foi considerável. Os poucos estudos que avaliaram os custos diretos e indiretos dessa doença não foram conduzidos no $\operatorname{Brasil}^{(4)}$. Ainda se deve considerar a cronicidade dessa condição e sua interferência na QV das mulheres, visto que uma doença crônica pode alterar a capacidade física $(67,6 \%)$, o trabalho/estudo/atividades do $\operatorname{lar}(64,8 \%)$ e a autoestima $(53,5 \%)$ dos indivíduos ${ }^{(5)}$.

Essa doença interfere significativamente na vida profissional, social e conjugal das portadoras, afetando sua $\mathrm{QV}^{(6)}$. Nesse sentido, é fundamental sua análise, com a finalidade de traçar estratégias que visem à promoção da saúde.

Desse modo, o presente estudo tem por objetivo avaliar a Qualidade de vida (QV) em pacientes com endometriose.

\section{MÉTODOS}

Realizou-se uma revisão bibliográfica buscando artigos científicos que descrevessem a relação entre endometriose e QV.

Selecionaram-se artigos publicados nos últimos 10 anos na base de dados PubMed utilizando os descritores quality of life and endometriosis e os seguintes filtros: artigos com abstract disponível, em humanos do sexo feminino, idioma inglês/português e cujos termos constavam no título/ abstract.

Realizou-se a busca em dezembro de 2013, a qual resultou em 147 artigos. Incluíram-se apenas artigos intimamente relacionados ao tema e que não avaliaram a contribuição do tratamento na QV. A seleção dos artigos foi baseada nos títulos e/ou resumos. Foram excluídos artigos do tipo revisão, relato de caso, carta ao editor, publicados antes de 2004, em idioma que não fosse inglês ou português, os que discutiam a influência do tratamento na QV de mulheres com endometriose e os que não tinham relação com o assunto da presente revisão.

Desse modo, apenas 12 artigos preencheram os critérios de elegibilidade.

\section{RESULTADOS E DISCUSSÃO}

A presente revisão incluiu 12 publicações sobre qualidade de vida $(\mathrm{QV})$ em pacientes com endometriose ${ }^{(7-18)}$, sendo um terço delas conduzido no Brasil ${ }^{(8,10,12,15)}$.

A avaliação da QV envolve aspectos multidimensionais, uma vez que ela não é caracterizada pela ausência de doença, e sim pelo bem-estar biopsicossocial, pelo como as pessoas vivem, sentem e compreendem seu cotidiano. São considerados os sentimentos, a cultura e o meio em que o indivíduo vive.

Esse capítulo será dividido em três tópicos: 1) QV em endometriose; 2) Fatores que interferem na QV e estratégias para promoção da saúde; e 3) Políticas públicas no Brasil para assistência a pacientes com endometriose. 


\section{QV em endometriose}

Três estudos empregaram o WHOQOL-bref (World Health Organization Quality of Life Assessement Instrumento de Avaliação de QV da OMS versão abreviada) para análise de $\mathrm{QV}^{(7-9)}$. Esse instrumento avalia quatro domínios: físico, psicológico, relação social e ambiente.

Os resultados de uma pesquisa ${ }^{(7)}$ revelaram que as mulheres classificaram sua QV como média nos domínios psicológico e social (escore mediano: 50). O escore do domínio físico foi ligeiramente abaixo da média (escore mediano: 48,2), ao passo que o domínio ambiental foi ligeiramente acima da média (escore mediano: 54,7). Quanto ao domínio físico, o mais prejudicado, os autores mostraram que a duração do tratamento e a intensidade da dor estavam correlacionadas com a redução da QV. Acrescenta-se a isso o baixo nível socioeconômico das mulheres avaliadas que eram provenientes do sistema público de saúde ${ }^{(7)}$.

Outro estudo $^{(8)}$ mostrou que os escores de QV foram similares entre os grupos com $(n=32)$ e sem $(n=25)$ endometriose (domínio físico: $54,2 \pm 12,8$ e $51,1 \pm 13,8, \mathrm{P}$ =0,504; domínio psicológico: 56,2 $\pm 14,4$ e $62,8 \pm 12,4, \mathrm{P}=$ 0,182; domínio social: $55,6 \pm 18,2$ e $62,1 \pm 19,1, \mathrm{P}=0,325$ e domínio ambiental: 59,2 $\pm 11,7$ e 61,2 $\pm 10,8, \mathrm{P}=0,608$, respectivamente). Pacientes com escores elevados de dor apresentaram redução na QV nas dimensões psicológica e ambiental, evidenciando uma influência negativa da dor na $\mathrm{QV}^{(8)}$.

A QV também foi avaliada em 134 mulheres, as quais foram divididas em três grupos: Grupo I - 49 pacientes com DPC e endometriose; Grupo II - 35 pacientes com DPC e sem endometriose; Grupo III - 50 pacientes saudáveis, com ausência de doenças ginecológicas ${ }^{(9)}$. A pontuação para $\mathrm{o}$ domínio físico ficou em 52,91; 44,71 e 68,68, p<0,001, para os grupos I, II e III, respectivamente. Seguem as pontuações para os demais domínios nos três grupos: psicológico - 57,14; 54,57 e 63,50, p 0,032; relações sociais - 61,22; 60,00 e $65,00, p=0,276$ e ambiente - 51,21; 47,14 e 52,13, $\mathrm{p}=0,279$. O escore WHOQOL-bref geral para os grupos I, II e III foi 55,64; 51,61 e 62,45, p=0,001, respectivamente.

Observaram-se diferenças significativas quando foram comparados os dois grupos de pacientes sintomáticos com o grupo controle nos domínios físico $(\mathrm{p}<0,001)$ e psicológico $(p=0,032)$. Houve também uma diferença significativa no escore WHOQOL-bref geral quando os grupos I e II foram comparados com o controle $(p=0,001)$. Não houve diferenças significantes entre os grupos quando se avaliou os domínios social e ambiental. Isso pode ser devido ao fato de que esses domínios não estão relacionados à doença ou ao sintoma especificamente, e sim ao estado socioeconômico e cultural da população estudada, atendida por um serviço de saúde pública no Brasil.
Quanto ao estado de saúde das pacientes, observou-se que, nos grupos I e II, aproximadamente $35 \%$ das pacientes descreveram sua saúde como sendo ruim ou muito ruim, contra apenas $2 \%$ no grupo controle $(p<0,001)$. Esse resultado seria esperado, tendo em vista que os grupos I e II são constituídos por pacientes com DPC.

Dois estudos mostraram que as pacientes com endometriose exibiram comprometimento significativo dos domínios físico e psicológico ${ }^{(7,9)}$. As questões referentes a esses domínios foram: - Domínio físico: 1) Dor e desconforto, 2) Energia e fadiga, 3) Sono e repouso, 9) Mobilidade, 10) Atividades da vida cotidiana, 11) Dependência de medicação ou de tratamentos e 12) Capacidade de trabalho; - Domínio psicológico: 4) Sentimentos positivos, 5) Pensar, aprender, memória e concentração, 6) Autoestima, 7) Imagem corporal e aparência, 8) Sentimentos negativos e 24) Espiritualidade/religião/crenças pessoais.

Sete estudos empregaram o SF-36 (Medical Outcomes Study 36 - Item Short-Form Health Survey) para avaliar $\mathrm{QV}^{(10-16)}$. Os escores para as oito categorias individuais do SF-36 são apresentados na Tabela I. Uma análise mais detalhada evidenciou que os domínios "dor", "vitalidade" e "saúde mental" apresentaram os menores escores. O primeiro domínio avalia o grau de limitação provocado pela dor; a vitalidade é referente ao grau de energia e disposição; e a saúde mental avalia como o indivíduo se sente na maior parte do tempo com relação a sentimentos de nervosismo, depressão, felicidade e paz. De um modo geral, independentemente do instrumento para mensuração da QV empregado, as habilidades psíquicas e físicas foram as mais comprometidas. As dimensões "aspectos físicos" (escore mediano 25) e "aspectos emocionais" (escore mediano 33,3) foram afetadas de modo significativo. Nenhuma correlação foi encontrada entre os parâmetros de QV e o nível de dor ${ }^{(10)}$.

Em outro estudo(12), as mulheres do grupo controle $(n=82)$ exibiram pontuação igual ou superior a 60 para todos os oito domínios individuais do SF-36, indicando uma boa QV (Tabela I). Entretanto, as pacientes com endometriose $(n=93)$ exibiram escores maiores que 60 apenas para os domínios "funcionamento físico" (70) e "aspectos emocionais" (67) - para os demais, os escores foram menores que 60 (Tabela I). A pontuação do PCS (Physical Component Sumary) foi 52,4 (50,3-53,6) no grupo controle e 40,3 (37,9-41,5) no grupo com endometriose. Já para MCS (Mental Component Sumary), obtiveram escore de $47,5(43,3-47,6)$ e $33,3(32,4-38,0)$ para os grupos controle e com endometriose, respectivamente. Diferenças entre PCS e MCS foram significativas $(\mathrm{p}<0,0001)^{(12)}$.

Uma redução na QV psíquica (MCS) do SF-36 ficou evidenciada em mulheres com endometriose no estádio avançado $^{(13)}$. Essa redução no MCS afeta o componente 





psíquico da paciente, e isso seria esperado em virtude da alta prevalência de ansiedade e depressão nas mulheres com endometriose $^{(7,19)}$.

Outro estudo ${ }^{(15)}$ revelou que o menor escore foi para o domínio "vitalidade" (média 51) e o maior foi para "capacidade funcional" (média 80) (Tabela I). Uma média aritmética considerando os escores das oito dimensões do SF-36 indicou um escore total equivalente a 62,6 . Os autores referiram que os escores de QV foram levemente superiores a de outros trabalhos publicados e atribuíram esse achado ao alto nível socioeconômico da população participante do estudo. O fato de essa população possuir maior renda permite acesso a medicamentos mais eficientes e ao tratamento médico. Embora esses aspectos não garantam cura ou remissão total dos sintomas, sem dúvida podem contribuir para melhorar a QV. Nesse trabalho, também se demonstrou que a intensidade da dor apresentou associação com menores escores em algumas escalas do SF36 (ex: capacidade funcional, aspectos físicos, dor, aspectos sociais). Em contrapartida, outro trabalho ${ }^{(16)}$ relatou escore total médio SF-36 de 50,68 $\pm 21,09^{(16)}$, inferior àquele apresentado previamente ${ }^{(15)}$.

Dois estudos investigaram o impacto da endometriose na $\mathrm{QV}$ e na produtividade no trabalho e demonstraram incapacidade significativa no PCS e MCS ${ }^{(14,17)}$. Um deles, do tipo multicêntrico, avaliou QV em 1.418 mulheres (745 com endometriose, 587 sintomáticas e 86 submetidas à esterilização laparoscópica) (Tabela I), cujas pontuações do PCS e MCS no grupo de endometriose foram 45,1 e 42,2, respectivamente. O outro estudo ${ }^{(17)}$ empregou o SF-12 e o EHP-5 (Endometriosis Health Profile), e os escores PCS e MCS do SF-12 foram 38,4 46,7 e 39,5 $\pm 6,2$, respectivamente. Com relação aos resultados do EHP-5, cerca de $40 \%$ das pacientes referiram que a dor controla suas vidas, causando prejuízo físico ou influenciando seu trabalho. As pacientes também apresentaram alterações na saúde emocional: aproximadamente $70 \%$ exibiram alterações no humor, $60 \%$ relataram que as pessoas não compreendem seus sentimentos e $48 \%$ perceberam mudanças em sua aparência física. A categoria "cuidado com crianças" foi irrelevante, tendo em vista que $64 \%$ das pacientes relataram não ter filhos e $56 \%$ delas referiram sentir-se deprimidas por esse motivo. Cerca de $40 \%$ relataram impacto na vida sexual e frustração frente à ineficácia dos tratamentos disponíveis. No domínio "relacionamento com seu médico", apenas 13\% comentaram que eles minimizam os sintomas associados à doença ${ }^{(17)}$.

Quanto à infertilidade, ela traz ao casal um ônus físico, psicológico, emocional e financeiro, e é mais impactante para as mulheres, pois interfere na concretização da maternidade ${ }^{(20)}$. Outro fator que pode provocar alterações psicológicas nas mulheres com endometriose é o atraso no diagnóstico, usualmente realizado por um procedimento cirúrgico denominado videolaparoscopia. Devido aos altos custos da laparoscopia em instituições privadas, muitas mulheres com endometriose procuram hospitais públicos, resultando em longas filas de espera para consultas agendadas em centros especializados e contribuindo diretamente para o atraso no diagnóstico ${ }^{(21,22)}$. A demora na indicação da videolaparoscopia pode levar ao diagnóstico tardio e ao comprometimento do futuro reprodutivo dessas mulheres, portanto, é necessária a detecção precoce dessa condição, principalmente em mulheres jovens ${ }^{(4)}$.

Outra pesquisa multicêntrica avaliou QV em 909 mulheres com endometriose atendidas em 12 centros terciários de saúde, provenientes de dez países ${ }^{(18)}$. Utilizouse o instrumento genérico EuroQol-5D, que avalia cinco dimensões, as quais, em conjunto, representam o estado de saúde. Os problemas referidos foram: mobilidade (16\%), autocuidado (3\%), atividades diárias (29\%), dor/desconforto $(56 \%)$ e ansiedade/depressão $(36 \%)^{(18)}$. Mulheres com endometriose exibiram um cálculo de anos de vida ajustado pela qualidade/QALYs (Quality-Adjusted Life Years) média de 0,809 no curso de um ano, o que representa uma redução de $19 \%$ na QV, quando comparado com uma pessoa com o melhor estado de saúde possível. O valor aproximado de 0,81 foi pior que $0,85-0,94$ QALYs por ano, observado para a população geral. Apenas $24 \%$ das mulheres apresentaram QALYs de 1,0, indicando que elas tinham a mesma QV que uma pessoa com o melhor estado possível de saúde. Duas mulheres exibiram valores de QALYS negativo, mostrando que elas consideravam seu estado atual de saúde pior do que a morte. Matematicamente, o QALY é calculado como a soma do produto de anos de vida e a QV em cada um desses anos ${ }^{(18)}$.

Os estudos apresentados mostraram que as pacientes com endometriose exibem redução na $\mathrm{QV}$, no entanto, não propõem estratégias para melhora dessa variável. A busca pela promoção da saúde vem ao encontro da seguinte afirmativa: "dar QV não significa somente acrescentar anos às vidas dos sujeitos, mas adicionar vida aos anos"(23).

\section{Fatores que interferem na $Q V$ e estratégias para promoção da saúde}

O alcance da QV envolve saúde, educação, trabalho, bem-estar emocional e interação social. Desse modo, alguns fatores podem estar contribuindo para a melhora da QV dessa mulheres, como melhor acesso à saúde, nível socioeconômico mais elevado, alto grau de escolaridade, hábitos saudáveis, realização e satisfação profissional, equilíbrio físico e psicológico. Outros fatores que influenciam negativamente a $\mathrm{QV}$ incluem mercado de trabalho competitivo, menor favorecimento econômico, estilo de vida, estado de saúde etc. 
Ao analisar QV, deve-se considerar que existem fatores que afetam positiva ou negativamente essa variável, e estes devem ser avaliados. Dos 12 artigos, apenas cinco deles correlacionaram QV com nível socioeconômico, dor e hábitos saudáveis ${ }^{(7-10,15)}$. Quatro estudos ${ }^{(7,9,10,15)}$ comentaram a influência do nível socioeconômico na QV das pacientes por eles estudadas. Em três deles, as pacientes eram originárias de serviços públicos de saúde e exibiam baixo nível socioeconômico ${ }^{(7,9,10)}$. Em outro estudo ${ }^{(15)}$, as pacientes exibiram alto nível socioeconômico, o que contribuiu positivamente para alcançar uma elevação na QV.

Uma possível explicação para isso pode ser o nível de escolaridade apresentado pelas pacientes, pois $87 \%$ possuíam o terceiro grau completo. Com relação aos diferentes níveis econômicos, mulheres de baixa renda têm piores condições de saúde, trabalho e lazer. No entanto, aquelas de maior poder aquisitivo geralmente estão inseridas em mercados de trabalho altamente competitivos e que demandam cada vez mais qualificação, portanto, elas lidam com a estressante incumbência de ascender profissional e economicamente sem deixar de lado sua vida pessoal. Quanto a este último aspecto, foi observado que $75 \%$ das mulheres são casadas, portanto, apresentam obrigações familiares, além das profissionais ${ }^{(15)}$.

Quatro estudos avaliaram a associação entre dor e $\mathrm{QV}^{(7,8,10,15)}$ e dois mostraram que pacientes com escores elevados de dor exibiam redução da $\mathrm{QV}^{(7,8)}$. Em endometriose, a dor é um sintoma frequente e preocupante. Uma diminuição nos sintomas dolorosos contribuiria para elevação da $\mathrm{QV}$, pois esta está relacionada ao nível de dor. Nesse sentido, seria conveniente e necessário desenvolver abordagens para gestão da dor. Um estudo conduzido em 2010 mostrou que a acupuntura promoveu uma redução estatisticamente significante na dor associada à endometriose, com consequente melhora na $\mathrm{QV}$ das pacientes ${ }^{(24)}$. Outra modalidade seria a terapia cognitivocomportamental, definida como uma abordagem psicológica que visa diminuir a dor ${ }^{(25)}$.

Um relato de caso(26) mostrou que o tratamento multidisciplinar que abrangeu o uso de fármacos, inativação dos pontos-gatilho (infiltração com anestésicos, agulhamento seco, acupuntura), fisioterapia, reeducação postural e suporte psicossocial proporcionou melhora significativa da dor e da QV de uma paciente de 45 anos que exibia DPC há seis anos. Esses trabalhos ${ }^{(24,25,26)}$ mostraram a importância de profissionais da fisioterapia e psicologia para o tratamento de pacientes com endometriose. Além da DPC, a dor durante o intercurso sexual afeta a sexualidade das pacientes. A sexualidade humana é considerada pela OMS como uma das dimensões da QV. Nessa direção, é necessário que a atividade sexual proporcione à paciente com endometriose um bem-estar físico e psicológico ${ }^{(17)}$.
Outro fator que contribui para a elevação da QV é a prática de exercícios físicos. Apenas $22,8 \%$ das mulheres com endometriose praticavam exercícios físicos e exibiram maiores escores na dimensão de saúde mental que aquelas que não praticavam $(\mathrm{p}<0,001)^{(10)}$. $\mathrm{O}$ maior nível de atividade física esteve associado à melhor percepção de QV em idosos, adultos aparentemente saudáveis ou em diferentes condições de saúde "(27); e os domínios individuais do SF-36 - "função física", "vitalidade" e "saúde mental" apresentaram concordância de $100 \%$ e $80 \%$, evidenciando associação positiva entre atividade física com QV, ou seja, a prática de exercícios estava relacionada com melhora significativa nesses domínios ${ }^{(27)}$.

De um modo geral, os estudos apresentados na Tabela 1 mostraram que os domínios "dor", "vitalidade" e "saúde mental" apresentaram os menores escores. Nessa direção, seria conveniente a inclusão da atividade física no tratamento da endometriose, com a finalidade de melhorar esses domínios específicos do SF-36. No entanto, a literatura científica é inconclusiva quanto aos benefícios dos exercícios físicos para pacientes com endometriose ${ }^{(28)}$. Estudos realizados nos Estados Unidos indicaram que a prática sistemática do exercício físico mostrou associação com ausência ou poucos sintomas depressivos ou de ansiedade $^{(29)}$. Sintomas depressivos pela ausência de filhos e ansiedade/depressão foram observados em $56 \%$ e $36 \%$, respectivamente, das mulheres com endometriose investigadas $^{(17,18)}$. Os distúrbios psicológicos podem comprometer a QV das pacientes, portanto, é recomendável a prática de atividade física. Os exercícios físicos também foram capazes de reduzir a dor e outros sintomas da fibromialgia, caracterizada por dor crônica generalizada ${ }^{(30)}$. Desse modo, a prática de exercícios físicos constitui uma intervenção de baixo custo e deveria ser indicada no tratamento de pacientes com endometriose, em virtude de promover uma melhora nos aspectos psicológicos e alívio da dor crônica, contribuindo efetivamente para elevação da QV de pacientes com essa doença ginecológica.

No entanto, o ato de exercitar-se precisa estar incorporado não somente ao cotidiano das pessoas, mas também à cultura popular, aos tratamentos médicos, ao planejamento da família e à educação infantil. Esses dados reforçam a necessidade de estímulos a programas de incentivo à prática de atividade física por políticas públicas $^{(31)}$.

Segundo a literatura ${ }^{(32)}$, a prática de atividade física pode ser realizada em espaços públicos de lazer. Estes constituem ambientes propícios para a promoção da saúde, devido à função de revitalizar e promover o bem-estar no meio urbano, possibilitando que as pessoas adquiram hábitos saudáveis, contribuindo nos aspectos emocionais, sociais, físicos e culturais dos indivíduos. A prática de 
exercícios tem sido considerada tratamento de primeira linha em diversas doenças crônicas, como diabetes do tipo 2, hipertensão arterial, osteoartrite, osteoporose, obesidade e câncer ${ }^{(33)}$. É considerada um potente fator de promoção da saúde e cabe ao profissional de Educação Física a (re) inserção dela na vida das pessoas, uma vez que é barata, segura e é capaz de reduzir sobremaneira a necessidade de medicamentos.

Uma pesquisa conduzida em Pelotas-RS mostrou que a realização de atividade física promoveu uma redução significativa nos gastos com saúde ao Sistema Único de Saúde (SUS) para tratamento de doenças crônicas. Os autores sugerem que ela seja oferecida de forma mais consistente à população brasileira e recomendam maior participação dos profissionais de Educação Física no SUS, como nos Núcleos de Apoio a Saúde da Família (NASFs), bem como um aumento no número de programas de promoção da atividade física ${ }^{(34)}$. Infelizmente, na atual revisão, não há estudos sobre atividade física e seus efeitos na endometriose em relação a controle dos sintomas, aspectos psicológicos e sua influência na QV.

Em suma, a endometriose é uma doença ginecológica crônica caracterizada por quadros álgicos significativos e com sintomas consideravelmente comprometedores para o cotidiano das mulheres que sofrem com esta afecção. Nesse sentido, é importante a busca por estratégias para promoção da saúde. Esta, segundo a Carta de Ottawa, deve ser entendida como a capacitação das pessoas e das comunidades para modificar os determinantes da saúde em benefício da própria $\mathrm{QV}$, resultando em uma perícia natural no controle desse processo ${ }^{(35)}$. Isso vem ao encontro da definição de saúde que não se caracteriza apenas como um estado de ausência de doenças nos indivíduos, mas busca um equilíbrio nos diferentes aspectos e sistemas que caracterizam o homem biológico, psicológico, social, emocional, mental e intelectual, resultando em sensação de bem-estar ${ }^{(31)}$.

\section{Políticas públicas no Brasil para assistência à pacientes com endometriose}

No Brasil, de acordo com o Ministério da Saúde, estima-se que de 7 a 10 milhões de mulheres tenham endometriose. Devido à alta prevalência, etiopatogenia incerta, cronicidade e morbidade, ela é considerada um problema de saúde pública. Por esse motivo, em 2006, o Ministério da Saúde do Brasil estabeleceu o Protocolo Clínico e as Diretrizes Terapêuticas para o Tratamento da Endometriose, pela Portaria SCTIE/MS n ${ }^{\circ}$ 69, de 06 de novembro de $2006^{(36)}$, que foi revisado e atualizado pela Portaria SAS/MS n ${ }^{\circ} 144$, de 31 de março de $2010^{(37)}$. No entanto, o tratamento medicamentoso ou cirúrgico proposto nesse protocolo visa à diminuição da dor e eliminação dos focos endometrióticos, promovendo tratamento dos sintomas físicos. Mas ainda há a necessidade de suporte psicológico para o tratamento da ansiedade e depressão apresentadas pelas mulheres, visando o reestabelecimento de sua QV.

Considerando que a endometriose é um problema que afeta a saúde da mulher, vale a pena se comentar as políticas públicas voltadas para essa população no Brasil. O Ministério da Saúde implantou, nos anos de 1984 e 2004, o Programa de Atenção Integral à Saúde da Mulher (PAISM) (38) e a Política Nacional de Atenção Integral à Saúde da Mulher (PNAISM) ${ }^{(39)}$, respectivamente. O PAISM, além de propiciar uma melhoria no atendimento ao período gravídicopuerperal, estabelece atenção a outras necessidades da saúde feminina, como as doenças de transmissão sexual e as ginecológicas malignas, tirando o foco de atenção à saúde da mulher somente na reprodução e maternidade. Já o PNAISM teve por finalidade atender melhor as questões já propostas pelo PAISM e o atendimento a grupos até então alijados da sociedade e áreas antes pouco assistidas.

O objetivo específico 1 da PNAISM é ampliar e qualificar a atenção clínico-ginecológica, inclusive para as portadoras da infecção pelo HIV e outras DSTs; além disso, há as seguintes metas: 1) reduzir a morbimortalidade por doenças crônico-degenerativas na população feminina, especialmente por doenças cardiovasculares e diabetes mellitus; 2) reduzir a morbimortalidade por Aids na população feminina; e 3) implantar a atenção qualificada às mulheres com queixas clínico-ginecológicas. Apesar de $\mathrm{o}$ atendimento às mulheres com doenças crônicas e queixas clínico-ginecológicas estar previsto no PNAISM (2004), não há referência à endometriose ${ }^{(38-40)}$. Considerando que a endometriose é uma doença complexa e prevalente, seria adequado um atendimento holístico às pacientes por meio de um tratamento multidisciplinar.

Portanto, o médico especialista, juntamente com um psicólogo ou profissional de saúde mental, enfermeiro, terapeuta sexual, fisioterapeuta e educador físico, poderia, em conjunto, orientar e traçar estratégias a serem alcançadas a curto, médio e longo prazo para controle dos sintomas da doença. Desse modo, a avaliação da QV em pacientes com endometriose poderia ser utilizada como um indicador prognóstico da melhora clínica.

\section{CONCLUSÃO}

Mulheres com endometriose apresentam significativa redução em sua qualidade de vida, influenciada pelos aspectos físicos e psicológicos, entre outros. 


\section{REFERÊNCIAS}

1. Giudice LC, Kao LC. Endometriosis. Lancet. 2004;364(9447):1789-99. Review.

2. Study protocol for the World Health Organization project to develop a Quality of Life assessment instrument (WHOQOL). Qual Life Res. 1993;2(2): 153-9.

3. Colwell HH, Mathias SD, Pasta DJ, Henning JM, Steege JF. A health-related quality-of-life instrument for symptomatic patients with endometriosis: a validation study. Am J Obstet Gynecol. 1998;179(1):47-55.

4. Spigolon DN, Amaral VF, Barra CMC. Endometriose: impacto econômico e suas perpectivas. Femina. 2012;40(3):129-34.

5. Martins LM, França AP, Kimura M. Quality of life of persons with chronic illness. Rev Latinoam Enferm. 1996;4(3):5-18.

6. Moradi M, Parker M, Sneddon A, Lopez V, Ellwood D. Impact of endometriosis on women's lives: a qualitative study. BMC Womens Health. 2014;14:123.

7. Sepulcri RP, Amaral VF. Depressive symptoms, anxiety, and quality of life in women with pelvic endometriosis. Eur J Obstet Gynecol Reprod Biol. 2009;142(1):53-6.

8. Souza CA, Oliveira LM, Scheffel C, Genro VK, Rosa V, Chaves MF, et al. Quality of life associated to chronic pelvic pain is independent of endometriosis diagnosis--a cross-sectional survey. Health Qual Life Outcomes. 2011;9:41.

9. Tripoli TM, Sato H, Sartori MG, Araujo FF, Girão MJ, Schor E. Evaluation of quality of life and sexual satisfaction in women suffering from chronic pelvic pain with or without endometriosis. J Sex Med. 2011;8(2):497-503.

10. Marques A, Bahamondes L, Aldrighi JM, Petta CA. Quality of life in Brazilian women with endometriosis assessed through a medical outcome questionnaire. J Reprod Med. 2004;49(2):115-20.

11. Laursen BS, Bajaj P, Olesen AS, Delmar C, ArendtNielsen L. Health related quality of life and quantitative pain measurement in females with chronic nonmalignant pain. Eur J Pain. 2005;9(3):267-75.

12. Petrelluzzi KF, Garcia MC, Petta CA, GrassiKassisse DM, Spadari-Bratfisch RC. Salivary cortisol concentrations, stress and quality of life in women with endometriosis and chronic pelvic pain. Stress. 2008;11(5):390-7.
13. Siedentopf F, Tariverdian N, Rücke M, Kentenich $\mathrm{H}$, Arck PC. Immune status, psychosocial distress and reduced quality of life in infertile patients with endometriosis. Am J Reprod Immunol. 2008;60(5): $449-61$.

14. Nnoaham KE, Hummelshoj L, Webster P, d'Hooghe $\mathrm{T}$, Nardone $\mathrm{FC}$, Nardone $\mathrm{CC}$, et al. Impact of endometriosis on quality of life and work productivity: a multicenter study across ten countries. Fertil Steril. 2011;96(2):366-373.e8

15. Minson FP, Abrão MS, Sardá Júnior J, Kraychete DC, Podgaec S, Assis FD. Importance of quality of life assessment in patients with endometriosis. Rev Bras Ginecol Obstet. 2012;34(1):11-5.

16. Montanari G, Di Donato N, Benfenati A, Giovanardi G, Zannoni L, Vicenzi C, et al. Women with deep infiltrating endometriosis: sexual satisfaction, desire, orgasm, and pelvic problem interference with sex. J Sex Med. 2013;10(6):1559-66.

17. Fourquet J, Báez L, Figueroa M, Iriarte RI, Flores I. Quantification of the impact of endometriosis symptoms on health-related quality of life and work productivity. Fertil Steril. 2011;96(1):107-12.

18. Simoens S, Dunselman G, Dirksen C, Hummelshoj L, Bokor A, Brandes I, et al. The burden of endometriosis: costs and quality of life of women with endometriosis and treated in referral centres. Hum Reprod. 2012;27(5):1292-9.

19. Lorençatto C, Vieira MJN, Pinto CLB, Petta CA. Avaliação da frequência de depressão em pacientes com endometriose e dor pélvica. Rev Assoc Med Bras 2002;48(3):217-21.

20. Monga M, Alexandrescu B, Katz SE, Stein M, Ganiats T. Impact of infertility on quality of life, marital adjustment, and sexual function. Urology. 2004;63(1):126-30.

21. Arruda MS, Petta CA, Abrão MS, Benetti-Pinto CL. Time elapsed from onset of symptoms to diagnosis of endometriosis in a cohort study of Brazilian women. Hum Reprod. 2003;18(4):756-9.

22. Petta CA, Matos AM, Bahamondes L, Faúndes D. Current practice in the management of symptoms of endometriosis: a survey of Brazilian gynecologists. Rev Assoc Med Bras. 2007;53(6):525-9.

23. Fleck MPA, Leal OF, Louzada S, Xavier M, Chachamovich E, Vieira G, et al. Desenvolvimento da versão em português do instrumento de avaliação 
de Qualidade de Vida da OMS (WHOQOL-100). Rev Bras Psiquiatr. 1999;21(1):19-28.

24. Rubi-Klein K, Kucera-Sliutz E, Nissel H, Bijak M, Stockenhuber D, Fink $\mathrm{M}$, et al. Is acupuncture in addition to conventional medicine effective as pain treatment for endometriosis? A randomised controlled cross-over trial. Eur J Obstet Gynecol Reprod Biol. 2010;153(1):90-3.

25. Mendes N, Figueiredo B. Psychological approach to endometriosis: women's pain experience and quality of life improvement. Psic Saúde \& Doenças. 2012;13(1):36-48.

26. Zakka TRM, Yeng LT, Teixeira MJ, Rosi Júnior J. Dor pélvica crônica não visceral: tratamento multidisciplinar. Relato de caso. Rev Dor. São Paulo, 2013;14(3):231-3.

27. Moura GC, Pucci F, Rech CR, Fermino RC, Reis RS. Associação entre atividade física e qualidade de vida em adultos. Rev Saúde Pública. 2012;46(1):166-79.

28. Bonocher CM, Montenegro ML, Rosa E Silva JC, Ferriani RA, Meola J. Endometriosis and physical exercises: a systematic review. Reprod Biol Endocrinol. 2014;12:4.

29. Mello MT, Boscolo RA, Esteves AM, Tufik S. O exercício físico e os aspectos psicobiológicos. Rev Bras Med Esporte. 2005;11(3):203-7.

30. Valim V. Benefícios dos exercícios físicos na fibromialgia. Rev Bras Reumatol. 2006; 46(1):49-55.

31. Araújo DSMS, Araújo CGS. Aptidão física, saúde e qualidade de vida relacionada à saúde em adultos. Rev Bras Med Esporte 2000; 6(5):194-203.

32. Costa da Silva EAP, Costa da Silva PP, Santos ARM, Cartaxo HGO, Rechia S, Freitas CMSM. Espaços Públicos de Lazer na Promoção da Qualidade de Vida: uma Revisão Integrativa. Licere, Belo Horizonte 2013; 16(2):1-18.

33. Gualano B, Tinucci T. Sedentarismo, exercício físico e doenças crônicas. Rev Bras Educ Fís Esporte. 2011;25(Num esp):37-43.
34. Bielemann RM, Knuth AG, Hallal PC. Atividade física e redução de custos por doenças crônicas ao Sistema Único de Saúde. Rev Bras Ativ Fís Saúde. 2010;15(1):9-14.

35. Ministério da Saúde (BR), Secretaria de Políticas de Saúde. Projeto Promoção da Saúde: as cartas da Promoção da Saúde. Brasília: Ministério da Saúde; 2002.

36. Ministério da Saúde (BR). Portaria SCTIE/MS n. 69, de 01 de novembro de 2006. Brasília: Ministério da Saúde; 2006.

37. Ministério da Saúde (BR). Portaria SAS/MS n. 144, de 31 março de 2010. Brasília: Ministério da Saúde; 2010.

38. Ministério da Saúde (BR). Assistência integral à saúde da mulher: bases da ação programática. Brasília: Ministério da Saúde; 1984.

39. Ministério da Saúde (BR). Política nacional de atenção integral à saúde da mulher: princípios e diretrizes. Brasília: Ministério da Saúde; 2004.

40. Freitas GL, Vasconcelos CTM, Moura ERF, Pinheiro AKB. Discutindo a política de atenção à saúde da mulher no contexto da promoção da saúde. Rev Eletrônica Enferm. 2009;11(2):424-8.

\section{Endereço primeiro autor:}

Maria Paula Custódio Silva

Universidade Federal do Triângulo Mineiro

Instituto de Ciências Biológicas e Naturais

Campus I

Praça Manoel Terra, 330

CEP: 38015-050 - Uberaba - MG - Brasil

E-mail: maria_paulacs@hotmail.com

\section{Endereço para correspondência:}

Alessandra Bernadete Trovó de Marqui Universidade Federal do Triângulo Mineiro

Instituto de Ciências Biológicas e Naturais

Campus I

Praça Manoel Terra, 330

CEP: 38015-050 - Uberaba - MG - Brasil

E-mail: alessandratrovo@hotmail.com 